\title{
Partial Fractional Differential Equations of Creeping and Vibrations of Plate and Their Solutions (First Part)
}

\author{
Katica (Stevanovic) Hedrih \\ Faculty of Mechanical Engineering University of Nis, Institute of Mathematics SANU Belgrade \\ Yu-18000 - Nis, ul. Vojvode Tankosica 3/22, SCG, Yugoslavia \\ katica@masfakni.ac.yu*khedrih@eunet.yu* \\ 0638757599
}

\section{SUMMARY}

Partial fractional-differential equations of creeping and vibrations of a plate are derived. A fractionaldifferential operator with creep material parameters is introduced. Plate material creeps and a constitutive relation of the stress-strain state is expressed through fractional derivatives. By using Navier's equations of quasi-statical equilibrium, stress component distributions in the points of the cross-section of the plate are derived as a function of coordinates of plate points.

An equation for the deformed middle surface of the plate has been derived for the case of plate characteristic-free oscillations. For that case, by using a numerical experiment over the solution of the fractional-differential equation $\quad \ddot{T}(t)+\left\{\omega_{0 m n}^{2}+\omega_{\alpha m n}^{2} D_{t}^{\alpha}\right\}[\mathbf{T}(t)]=0$, time-function surfaces $\mathbf{T}_{m n}\left(t, \omega_{0 m n}, \omega_{\alpha m n}, \alpha\right)$ have been constituted as visualizations used for expressing the creeping properties of the plate.

Keywords: Plate, creep, fractional derivative, vibrations, partial fractional-differential equation.

\section{INTRODUCTION}

The mechanics of a hereditary medium (material) is presented in scientific literature by an array of fundamental monographs and is widely used in engineering analyses of strength and deformability of constructions made of new construction materials. The relevance of that direction of development of mechanics is conditioned by engineering practice by utilizing the new construction materials on a synthetic base, the mechanical properties of which often have a distinct creep of rheological character.

The paper $/ 4$ / by Hedrih contains results of transversal vibrations of a prismatic beam of a fractional order 
derivative constitutive-relation beam. In the paper / $5 /$ by Hedrih and Filipovski, longitudinal creep vibrations of a fractional-order derivative rheological rod with a variable cross section are examined. For the case of free creep vibrations, the eigenfunction and time function are determined for different examples of boundary conditions. Different boundary conditions are analyzed and a series of eigenvalues and characteristic circular frequencies of longitudinal creep vibrations, as well as tables of these values, are completed.

The discrete continuum method for examples of homogeneous discrete systems with a limited number of degrees of motion freedom dynamics is defined by Hedrih $/ 6 /$. These systems are in the form of homogeneous chains and nets in space and plane, and material points of these nets and chains are tied by elastic, standard hereditary or creep elements of a fractional-order derivative constitutive stress-strain relation. A light standard creep element is defined by a constitutive relation of a stress-strain state, for the creation of which fractional order derivatives were used. An integral theory of analytical dynamics of discrete hereditary systems is presented in the monograph $/ 3 /$ by Gorosko and Hedrih.

In $/ 1 /$ and $/ 2 /$ fractional calculus is mathematically based on a fractional-order derivative of corresponding integral and differential equations.

\section{BASIC SUPPOSITIONS AND CONSTITUTIVE RELATION OF THE STRESS AND STRAIN STATE OF THE PLATE}

Let us suppose that a plate is thin and there is no deplanation of the cross sections in the conditions of the creep material $/ 7 /$. Also, we suppose that cross sections are always orthogonal with respect to the middle plane of the plate. If a thin plate is creep-bent with a small deflection, i.e., when the deflection of the middle surface is small compared with the thickness $h$, the following assumption can be made: $1^{*}$ The orthogonal material lines to the middle surface before creep bending are deformed into orthogonal material lines of the middle surface after bending. 2* The stress $\sigma_{z}$ is small compared with the other stress components and may be neglected in the stress strain relations. $3^{*}$ The middle surface remains unstrained after bending.

On the basis of the foregoing, we suppose that the displacements $u(x, y, z, t)$ and $v(x, y, z, t)$ of the point $N(x, y, z)$ in the direction of the coordinate axes $x$ and $y$, can be expressed as functions of its distance $z$ from the plate middle surface and its transversal displacement $w(x, y, t)$ in direction of the axis $z$, and the same displacement of the corresponding point $N_{0}(x, y, 0)$ in the plate middle surface, as in $/ 8 /$ by Raskovic.

Let us suppose the following relations between stresses and strains, in the plate's stressed and strained material with creep properties:

$$
\begin{aligned}
& \sigma_{x}=\mathbf{E}_{0 x} \varepsilon_{x}^{x}(t)+\mathbf{E}_{\alpha x} \mathcal{D}_{t}^{\alpha_{i}}\left[\varepsilon_{x}^{x}(t)\right] \\
& \sigma_{y}=\mathbf{E}_{0 y} \varepsilon_{y}^{y}(t)+\mathbf{E}_{\alpha y} \mathcal{D}_{t}^{\alpha_{t}}\left[\varepsilon_{y}^{y}(t)\right] \\
& \tau_{x y}=\mathbf{G}_{0} \gamma_{x y}(t)+\mathbf{G}_{\alpha} \mathcal{D}_{t}^{\alpha_{x y}}\left[\gamma_{x y}(t)\right] \text { where: } \mathcal{D}_{t}^{\alpha}[\varepsilon(t)]=\frac{1}{\Gamma(1-\alpha)} \frac{d}{d t} \int_{0}^{t} \frac{\varepsilon(\tau)}{(t-\tau)^{\alpha}} d \tau
\end{aligned}
$$


a differential operator with a fractional-order derivative (see $/ 1 /$ and $/ 2 /$ ), defined by a material parameter $\alpha$ that satisfies the following condition: $0<\alpha<1$. In the above relations $\mathbf{E}_{a x}, \mathbf{E}_{0 y}, \mathbf{E}_{\alpha x}, \mathbf{E}_{a y}$ are the elasticity coefficients of loading the plate material, momentary and prolondeons, in the corresponding directions of the axes $x$ and $y ; \alpha_{x}, \alpha_{y}$ and $a_{x y}$ are the corresponding coefficients of the creep of plate material for axial and shearing loads and: $\mathbf{G}_{0}=\frac{\mathbf{E}_{0}}{2(1+\mu)}$ and $\mathbf{G}_{\alpha}=\frac{\mathbf{E}_{\alpha}}{2(1+\mu)}$ are the corresponding shear moduli.

Also, we introduce the resulting dilatations of the plate:

$$
\begin{aligned}
& \varepsilon_{x}=\varepsilon_{x}^{x}-\mu \varepsilon_{y}^{y} \quad \varepsilon_{y}=\varepsilon_{y}^{y}-\mu \varepsilon_{x}^{x} \\
& \varepsilon_{x}^{x}=\frac{\varepsilon_{x}+\mu \varepsilon_{y}}{1-\mu^{2}} \varepsilon_{y}^{y}=\frac{\varepsilon_{y}+\mu \varepsilon_{x}}{1-\mu^{2}}
\end{aligned}
$$

where $\mu$ is Poisson's coefficient of the transversal contractions.

On the basis of the previous suppositions and relations we can write the following constitutive stressstrain relations:

$$
\begin{aligned}
& \left.\sigma_{x}=\frac{\mathbf{E}_{0 x}}{\left(1-\mu^{2}\right)}\left(\varepsilon_{x}(t)+\mu \varepsilon_{y}(t)\right)+\frac{\mathbf{E}_{\alpha x}}{\left(1-\mu^{2}\right)} D_{t}^{\alpha_{x}} \mid \varepsilon_{x}(t)+\mu \varepsilon_{y}(t)\right] \\
& \sigma_{y}=\frac{\mathbf{E}_{0 y}}{\left(1-\mu^{2}\right)}\left(\tilde{c}_{y}(t)+\mu \varepsilon_{x}(t)\right)+\frac{\mathbf{E}_{\alpha y}}{\left(1-\mu^{2}\right)} \nu_{t}^{\alpha_{y}}\left[\varepsilon_{y}(t)+\mu \varepsilon_{x}(t)\right] \\
& \overline{-}_{x y}=\mathbf{G}_{0} \gamma_{x y}(t)+\mathbf{G}_{\alpha} \nu_{t}^{\alpha_{x y}}\left[\gamma_{x y}(t)\right]
\end{aligned}
$$

Now, we introduce into the above equations - the relation between stress components and strain components the expression of strain tensor components $/ 8 /$ expressed as transversal displacements $w(x, y, t)$ the corresponding point of the plate middle surface $N(x, y, 0)$ and the coordinate $z$ of the corresponding plate point $N(x, y, z)$. Then we obtain the relations between stress components and transversal displacement $w(x, y, t)$ in the following form:

$$
\begin{aligned}
& \sigma_{x}=-\frac{\mathbf{E}_{0 x} z}{\left(1-\mu^{2}\right)}\left(\frac{\partial^{2} w(x, y, t)}{\partial x^{2}} \div \mu \frac{\partial^{2} w(x, y, t)}{\partial y^{2}}\right)-\frac{\mathbf{E}_{\alpha x} z}{\left(1-\mu^{2}\right)}{\widetilde{\sigma_{t}}}_{t}^{\alpha_{t}}\left[\frac{\partial^{2} w(x, y, t)}{\partial x^{2}}+\mu \frac{\partial^{2} w(x, y, t)}{\partial y^{2}}\right] \\
& \sigma_{y}=-\frac{\mathbf{E}_{0 y^{2} z}}{\left(1-\mu^{2}\right)}\left(\frac{\partial^{2} w(x, y, t)}{\partial y^{2}} \div \mu \frac{\partial^{2} w(x, y, t)}{\partial x^{2}}\right)-\frac{\mathbf{E}_{\alpha y^{2}}}{\left(1-\mu^{2}\right)} \widetilde{\nu}_{t}^{\alpha}\left[\frac{\partial^{2} w(x, y, t)}{\partial y^{2}}+\mu \frac{\partial^{2} w(x, y, t)}{\partial x^{2}}\right] \\
& \tau_{x y}-\frac{z \mathbf{E}_{0}}{(1+\mu)} \frac{\partial^{2} w(x, y, t)}{\partial x \partial y}-\frac{z \mathbf{E}_{\alpha_{x y}}}{(1+\mu)} \tau_{t}^{\prime \prime x}\left[\frac{\partial^{2} w(x, y, t)}{\partial x \partial y}\right]
\end{aligned}
$$


These expressions show that the stress tensor components $\sigma_{x}, \sigma_{y}$ and $\tau_{x y}$ are linear functions of the coordinate $z$ of the corresponding plate point $N(x, y, z)$ and distributions of these stress components are the same as for a plate of pure elastic material.

For homogeneous and isotropic materials with equal parameters of material creep properties, $\alpha_{x}=\alpha_{y}=\alpha$; also, the coefficients of rigidity, momentary and prolondeons, are: $\mathbf{E}_{0 x}=\mathbf{E}_{0 y}=\mathbf{E}_{0}$ and $\mathbf{E}_{\alpha \alpha}=\mathbf{E}_{\phi}=\mathbf{E}_{\alpha}$ in all directions at the corresponding point

\section{NAVIER'S EQUATIONS OF QUASI-STATIC EQUILIBRIUM OF THE DEFORMABLE PLATE AND COMPONENTS OF THE PLATE STRESS STATE}

When quasi-static equilibrium conditions of forces applied to the plate elements are satisfied, it is necessary that Navier's equations /8/ of the equilibrium of every part of the deformable body be satisfied. By using previously derived expressions (1) of the stress state tensor components $\sigma_{x}, \sigma_{y}$ and $\tau_{x y}$ and introducing into Navier's equations of the equilibrium of every part of the deformable body, we obtain unknown stress state tensor components, $\tau_{x z}, \tau_{y z}$ and $\sigma_{z}$. By using boundary conditions on the contour surfaces parallel to the middle surface of the plate for $z=i \frac{h}{2}$, it is easy to obtain expressions of the unknown integral unknown functions.

Now, the expressions for the shear stress components $\tau_{x z}$ and $\tau_{y z}$ take the following form:

$$
\begin{aligned}
& \tau_{x z}(x, y, z, t)=\tau_{z x}(x, y, z, t)=-\frac{\left(h^{2}-4 z^{2}\right)}{8\left(1-\mu^{2}\right)}\left\{\mathbf{E}_{0} \frac{\partial}{\partial x} \Delta w(x, y, t)+\mathbf{E}_{\alpha} \nu_{t}^{\alpha}\left[\frac{\partial}{\partial x} \Delta w(x, y, t)\right]\right\} \\
& \tau_{y z}(x, y, z, t)=\tau_{z y}(x, y, z, t)=-\frac{\left(h^{2}-4 z^{2}\right)}{8\left(1-\mu^{2}\right)}\left\{\mathbf{E}_{0} \frac{\partial}{\partial y} \Delta w(x, y, t)+\mathbf{E}_{\alpha} D_{t}^{\alpha}\left[\frac{\partial}{\partial y} \Delta w(x, y, t)\right]\right\}
\end{aligned}
$$

From the obtained expressions we can see that distributions of the shear stresses $\tau_{x z}$ and $\tau_{y z}$ along plate depth are the same as in the case of an ideal elastic plate and are parabolic form functions of the $z$-axis direction point distance from middle plate surface.

The expression for the normal stress component $\sigma_{z}$ for the plane with a normal in the $z$-axis direction takes the following form:

$$
\sigma_{z}(x, y, z, t)=\frac{\left(3 h^{2} z-4 z^{3}-1\right)}{24\left(1-\mu^{2}\right)}\left\{\mathbf{E}_{0} \Delta \Delta w(x, y, t)+\mathbf{E}_{\alpha} D_{t}^{\alpha}[\Delta \Delta w(x, y, t)]\right\}+\rho g\left(\frac{h}{2}-z\right)
$$




\section{PARTIAL FRACTIONAL-DIFFERENTIAL EQUATION OF THE DEFORMABLE PLATE MIDDLE SURFACE}

By using the boundary equilibrium condition that the normal stress component $\sigma_{z}$, expressed by (2), for the upper plate surface be equal to the external normal surface loading $p(x, y)$, we can write the following:

$$
\left\{\Delta \Delta w(x, y, t)+\kappa_{\alpha} \mathcal{D}^{\alpha}[\Delta \Delta w(x, y, t)]\right\}=\frac{\ddot{p}(\ddot{\mu}, \dot{y})-\rho g h}{D_{0}}
$$

where for the cylindrical flexural rigidity, $D_{0}$ and $D_{\alpha}$, momentary and prolondeons, of the loading processes to the plate material with creeping properties, as well as $\kappa_{\alpha}$ as the ratio of these rigidities, are introduced in the following forms:

$$
\mathrm{D}_{0}=\frac{\mathbf{E}_{0}}{12\left(1-\mu^{2}\right)}, \quad \mathrm{D}_{\alpha}=\frac{\mathbf{E}_{\alpha}}{12\left(1-\mu^{2}\right)}, \quad \kappa_{\alpha}=\frac{\mathrm{D}_{\alpha}}{\mathrm{D}_{0}}=\frac{\mathbf{E}_{\alpha}}{\mathbf{E}_{0}} .
$$

The above partial fractional-differential Eq. (3) is the equation of the transversal quasi-static displacement $w(x, y, t)$ of the middle surface point $N(x, y)$, loaded by external plate surface transversal excitation $p(x, y)$ and external volume excitation $\rho g h$. We conclude that for obtaining the last previous partial fractionaldifferential Eq. (3) of the transversal displacement $w(x, y, t)$ of the middle surface point $N(x, y)$. loaded by external plate surface transversal excitation $p(x, y)$ and external volume excitation $p g h$, one could use the idea of Sophie Germain (1815), submitted as a memoir to the Paris Academy of Sciences and corrected by Lagrange.

If we introduce in this equation the specific volume inertia force $\operatorname{ch} \frac{\partial^{2} w(x, y, t)}{\partial t^{2}}$, then we obtain the corresponding partial fractional-differential equation of the transversal displacement oscillation $w(x, y, t)$ of the creeping plate middle surface, loaded by external plate surface transversal excitation $p(x, y, t)$, in the following form:

$$
\left\{\Delta \Delta w(x, y, t)+\kappa_{\alpha} D_{t}^{\alpha}[\Delta \omega(x, y, t)]\right\}=\frac{p(x, y, t)-\rho g h}{D_{0}}-\frac{\rho h}{D_{0}} \frac{\partial^{2} w(x, y, t)}{\partial t^{2}}
$$

In the above discussion, the plate was assumed to be bent at a small deflection by lateral (transversal) loads only. If there are forces acting in the middle surface of the plate in addition to the lateral loads, the above governed partial fractional differential Eq. (4) must be modified to take into account the effects of these in-surface forces, see $\mathbf{8} /$.

In the general case of an elementary block with edges $d x$ and $d y$ and depth $h$, excited by external 
transversal surface forces $p(x, y, t) d x d y$ and forces $\mathbf{X}^{\prime}, \mathbf{Y}^{\prime}$ and $\mathbf{Y}_{x}^{\prime}=\mathbf{X}_{y^{\prime}}$ in the plate middle surface, is excited by surface forces caused by the appearance of stresses, and their equivalent action as bending moments, moments of torsion and transversal forces. We can also calculate changes in these surface forces and moments caused by changing of the coordinates from $x$ and $y$ to $x+d x$ and $y+d y$. Also, we must calculate the change of the normal direction of the cross-sectional surface.

After introducing into the above Eq. (4) members corresponding to the influence of the external forces $\mathbf{X}^{\prime}, \mathbf{Y}^{\prime}$ and $\mathbf{Y}_{x}{ }^{\prime}=\mathbf{X}_{y}{ }^{\prime}$ in the plate middle surface, takaing into consideration dynamic equilibrium conditions in the $0 x$ and $0 y$ directions, we obtain the final form of the transversal oscillations equation of the thin plate of the creep material as follows:

$$
\left\{\left(1+\kappa_{\alpha} D_{t}^{\alpha}\right)[\Delta \Delta w(x, y, t)]\right\}=\frac{p(x, y, t)}{D_{0}}+\frac{1}{D_{0}}\left(\mathbf{X}^{\prime} \frac{\partial^{2} w}{\partial x^{2}}+\mathbf{Y}^{\prime} \frac{\partial^{2} w}{\partial y^{2}}+2 \mathbf{X}_{y}{ }^{\prime} \frac{\partial^{2} w}{\partial x \partial y}\right)-\frac{\rho h}{D_{0}} \frac{\partial^{2} w(x, y, t)}{\partial t^{2}}
$$

This equation is derived based on assumption that the own weight of the plate is neglected and that the depth of the plate is small.

\section{FREE PLATE CREEPING OSCILLATIONS}

By introducing the notation $c_{i}=\frac{D_{0}}{\rho h}=\frac{E_{g} h^{3}}{12 \rho h\left(1-\mu^{2}\right)}$, and by using the derived Eqs. (4), we can obtain the partial fractional-differential equation of the transversal oscillations of the free plate in the following form:

$$
\frac{\partial^{2} w(x, y, t)}{\partial t^{2}}+c_{0}^{4}\left\{\left(1+\kappa_{\alpha} D_{t}^{\alpha}\right)[\Delta \Delta w(x, y, t)]\right\}=0
$$

The solution of the above fractional derivative-partial-differential equation can be looked for in Bernoulli's method of particular integrals in the form of multiplication of two functions (see /8/ and /7/), where the first $\mathrm{W}(x, y)$ depends only on the space coordinates $x$ and $y$, and the second is a time function $T(t)$ :

$$
w(x, y, t)=\mathrm{w}(x, y) \mathrm{r}(t)
$$

The assumed solution (6) is introduced in the above Eq. (5) and by introducing the notation of the constants

$$
\omega_{0}^{2}=k^{4} c_{0}^{4}=k^{4} \frac{D_{0}}{\rho h}=k^{4} \frac{\mathbf{E}_{0} h^{2}}{12 \rho\left(1-\mu^{2}\right)} \text { and } \omega_{\alpha}^{2}=\kappa_{\alpha} \omega_{0}^{2}=k^{4} \kappa_{\alpha} c_{0}^{4}=k^{4} \frac{D_{\alpha}}{\rho h}=k^{4} \frac{\mathbf{E}_{\alpha} h^{2}}{12 \rho\left(1-\mu^{2}\right)}
$$


it is easy to divide the above equation in two as follows:

*first, a fourth-order partial differential equation on an unknown eigenfumction $\mathbf{w}(x, y)$ of space coordinates $x$ and $y$ in the form:

$\Delta \Delta \mathbf{W}(x, y)-k^{4} \mathbf{W}(x, y)=0$ or in the form of two second order differential equations: $\Delta \mathbf{W}(x, y) \pm k^{2} \mathbf{W}(x, y)=0$ and

* second, a fractional-differential equation on an unknown time function $T(t)$ :

$$
\ddot{\mathbf{T}}(t)+\omega_{0}^{2}\left(1+\kappa_{\alpha} D_{t}^{\alpha}\right)[\mathbf{T}(t)]=0
$$

If the plate is circular, then the general solution for the transversal plate middle surface point displacement has the following form (see /8/):

$$
w(r, \varphi, t)=\sum_{n=1}^{\infty} \sum_{m=1}^{\infty}\left[\mathbf{J}_{n}\left(k_{n m} r\right)+K_{n m} \mathbf{I}_{n}\left(k_{n m m} r\right)\right] \sin \left(n \varphi+\varphi_{0 n}\right) \mathbf{r}_{m n}(t),
$$

where $\mathbf{J}_{n}(x)$ is a Bessel's function of the first kind with a real argument, and $\mathbf{I}_{n}(x)$ is a modified Bessel's function of the first kind with imaginary arguments, where time functions $\mathbf{T}_{n m}(t)$ are in the form of a series (see $/ 1 /, / 2 /, / 4 /$ and $/ 5 /$ ):

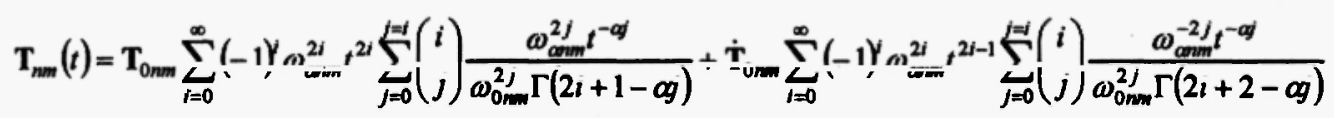

where we introduce the following notations:

$$
\omega_{0 n m}=k_{n m}^{2} c_{0}^{2}=k_{n m}^{2} \sqrt{\frac{D_{0}}{\rho h}}=k_{n m}^{2} \frac{h}{2} \sqrt{\frac{\mathbf{E}_{0}}{3 \rho\left(1-\mu^{2}\right)}}, \omega_{a n m}=\omega_{0 n m} \sqrt{\kappa_{\alpha}}=k_{n m}^{2} c_{0}^{2} \sqrt{\kappa_{\alpha}}=k_{n m}^{2} \sqrt{\frac{D_{\alpha}}{\rho h}}=k_{n m}^{2} \frac{h}{2} \sqrt{\frac{\mathbf{E}_{\alpha}}{3 \rho\left(1-\mu^{2}\right)}},
$$

and $k_{n m}$ is an own characteristic number defined as a series of roots of characteristic equations obtained from the corresponding plate boundary conditions.

The time functions $\mathbf{T}_{n m}(t)$ are defined as a solution of the corresponding ordinary fractional differential equation defined by (7).

\section{QUASI-STATIC TRANSVERSAL CREEP OF THE MIDDLE SURFACE RECTANGULAR PLATE WITH HINGED ENDS ALONG THE CONTOUR.}

For the study of the quasi-static transversal creep of the middle surface rectangular plate with $(a, b)$ and with hinged edges along the contour, we start with a partial fractional-differential equation loaded by a transversal surface distributed force $p(x, y)$, with a neglected inertial member, in the following form:

$$
\left\{\left(1+\kappa_{\alpha} \widetilde{D}_{t}^{\alpha}\right)[\Delta \Delta w(x, y, t)]\right\}=\frac{p(x, y)}{D_{3}}
$$


with accomplished corresponding boundary conditions (see /8/).

The above Eq. (9) is solved as follows:

$$
w(x, y, t)=\sum_{m=1}^{\infty} \sum_{n=1}^{\infty} \mathbf{T}_{m n}(t) \sin \frac{m \pi}{a} x \sin \frac{n \pi}{b} y
$$

where the time function $T_{n m}(t)$ of the quasi-static creep is the solution of the ordinary fractional-differential equation in the following form: $\left.\left(1+\kappa_{\alpha} \mathrm{D}_{i}^{\alpha}\right) \mathrm{T}_{m n}(t)\right]=\tilde{p}_{m n}$.

We obtain the transversal displacement $w(x, y, t)$ of the creep of the middle surface plate points for the quasi-static regime in the following form:

$$
w(x, y, t)=\frac{4}{D_{0} a b} \sum_{m=1}^{\infty} \sum_{n=1}^{\infty} \sum_{i=0}^{\infty} \frac{(-1)^{j} t^{a(t+1)}}{\kappa_{a}^{i+1} \Gamma[\alpha(i+1)+1]} \frac{\sin \frac{m \pi}{a} x \sin \frac{n \pi}{b} y}{\left[\left(\frac{m \pi}{a}\right)^{2}+\left(\frac{n \pi}{b}\right)^{2}\right]^{2}} \int_{0}^{a} \int_{0}^{b} p(x, y) \sin \frac{m \pi}{a} x \sin \frac{n \pi}{b} y d x d y
$$

\section{NUMERICAL EXPERIMENT AND RESULTS.}

By using the expression obtained for the time function $\mathrm{T}_{n m}(t)$ with corresponding particular solutions, we made a numerical experiment for characteristic cases and ratios of plate parameters, coefficient $\alpha$ of the creeping material, and the results are presented below in Figs. $1 a^{*}$ and $b^{*}$.
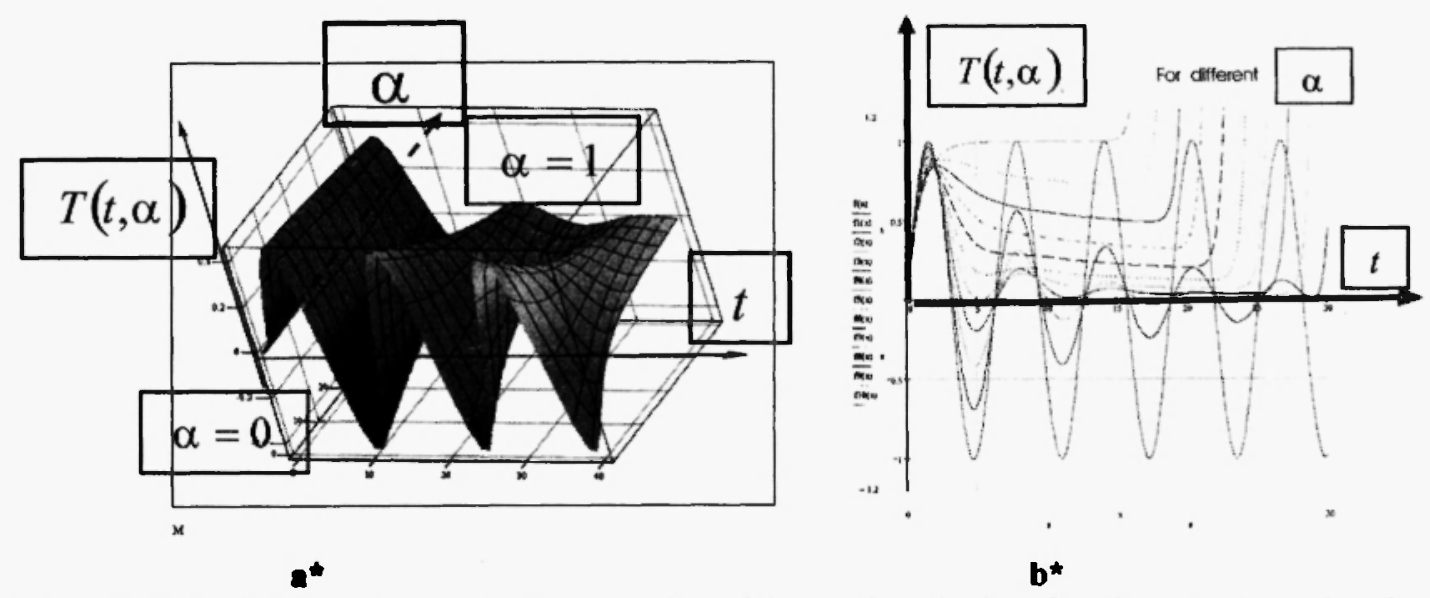

Fig. 1: Numerical simulations and graphical presentation of the results. $a^{\star}$ Time function $T(t, \alpha)$ surface for the plate transversal vibrations' kinetic and creep material parameters $\left(\frac{\omega_{m}}{\omega_{0 *}}\right)=1 ; b^{\star}$ Time functions $T(t, \alpha)$ - curve families for the different plate transversal vibrations kinetic and discrete values of the creep material parameters $0 \leq \alpha \leq 1: a^{\star}$ and $c^{\star}\left(\frac{\omega_{a x}}{\omega_{0 x}}\right)=1 ; b^{*}$ and $d^{\star}\left(\frac{\omega_{a x}}{\omega_{0 x}}\right)=\frac{1}{4} ; \mathrm{e}^{\star}\left(\frac{\omega_{a x}}{\omega_{0 x}}\right)=\frac{1}{3} ; \mathrm{f}^{\star}$ $\left(\frac{\omega_{a x}}{\omega_{0 x}}\right)=3$ 


\section{CONCLUDING REMARKS}

In this paper, a new theory of the deformation and oscillations of a thin creeping material plate for a small deformation is presented. Creeping properties of the material plate are presented by using constitutive relations in the fractional order differential stress-strain form with terms of a fractional order derivative with respect to time. By using the presented assumptions and the theory pointed out, the partial fractional differential equations of the quasi-static equilibrium of the plate and the transversal oscillations are derived and solved for different deformations and oscillations of the creeping state are obtained for some boundary plate conditions. Also, the expressions for the stress tensor component distributions in the plate of the creeping material are derived. The expressions for the bending and twisting moments and the transversal force are derived. From the obtained analytical and numerical results for free transversal creep vibrations of a fractional derivative order hereditary homogeneous thin plate, it can be seen that the fractional derivative order hereditary properties are in all cases convenient for changing the time function, depending on material creep parameters, and that fundamental eigen-fumction, depending on space coordinates is dependent only on the boundary conditions and geometrical properties of the plate.

\section{ACKNOWLEDGMENT}

Parts of this research were supported by the Ministry of Sciences, Technologies and Development of the Republic of Serbia through the Mathematical Institute SANU Belgrade Grants No. 1616, Real Problems in Mechanics, and the Faculty of Mechanical Engineering University of Nis Grant No. 1828, Dynamics and Control in Active Structures.

\section{REFERENCES}

1. M. Enelund, Fractional Calculus and Linear Viscoelasticity in Structural Dynamics, Division of Solid Mechanics, Chalmers Tekniska Hogskola, Goteborg, Sweden, 1996., p.1-27, A1-33+B1-20+C1-19+D128+E1-26.

2. R. Gorenflo and F. Mainardi, Fractional Calculus, Integral and Differential Equations of Fractional Order, CISM, Lecture Notes, Udine, Italy, Preprint, 54 pages, 2000; pp. 223-276.

3. Oleg Aleksandrovið Gorosko i Katica (Stevanovic) Hedrih, Analiticka dinamika (mehanika) diskretnih naslednih sistema, (Analytical Dynamics (Mechanics) of Discrete Hereditary Systems), University of Nis, 2001 , p. 426.

4. Katica (Stevanovic) Hedrih, Transversal Creep Vibrations of a Beam with Fractional Derivative Constitutive Relation Order, Proceedings of Fourth International Conference on Nonlinear Mechanics (ICNM-IV), 2002; pp. 584-595. 
5. K Hedrih (Stevanovic) and A. Filipovski, Longitudinal Creep Vibrations of a Fractional Derivative Order Rheological Rod with Variable Cross Section, Facta Universitatis, Series Mechanics, Automatics Control and Robotics, Vo. 3, No. 12, 2002, pp. 327-350. (in English). YU ISSN 0354-2009.

6. K. Hedrih (Stevanović), Discrete Continuum Method, Recent Advances in Analytical Dynamics Control, Stability and Differential Geometry, Proceedings Mathematical Institute SANU, 2002; pp. 30-57, 151, ISBN 86-80593-32-X.

7. V.S. Janković, V.P. Potić and K. Hedrih (Stevanović), Parcijalne diferencijalne jednačine $i$ integralne jednacine sa primenama $u$ inženjerstvu (Partial differential equations and integro-differential equations with examples in engineering), Univerzitet u Nišu, 1999, str. 347. (in Serbian).

8 D. Raškovic (1985), Teorija elastičnosti (Theory of Elasticity), Nau na knjiga, 1985, 414. 